Trauma Berufskrankh 2010 - 12 [Suppl 4]:416-420 DOI 10.1007/s10039-010-1636-5

Online publiziert: 6 . August 2010

(c) Springer-Verlag 2010
M. Frink ${ }^{1}$ - W. Koppert ${ }^{2} \cdot$ H. Andruszkow ${ }^{1} \cdot$ C. Krettek ${ }^{1} \cdot$ F. Hildebrand ${ }^{1}$

${ }^{1}$ Unfallchirurgische Klinik, Medizinische Hochschule Hannover

${ }^{2}$ Klinik für Anästhesiologie und Intensivmedizin, Medizinische Hochschule Hannover

\title{
Schnittstelle Schockraummanagement
}

Trotz deutlicher Verbesserungen der Behandlung von schwerstverletzten Patienten sind Unfälle noch immer für den höchsten Verlust an Lebensjahren im Kollektiv der unter 45-Jährigen verantwortlich und verursachen damit einen größeren sozioökonomischen Schaden als andere Erkrankungen. Bei etwa 6o\% der versterbenden polytraumatisierten Patienten tritt der Tod innerhalb der ersten $24 \mathrm{~h}$ ein. Deshalb ist ein schnelles kompetentes Management im Schockraum von großer Bedeutung.

Aufgrund der vielfältigen Möglichkeiten liegen die Herausforderungen bei der Behandlung polytraumatisierter Patienten nicht mehr in den einzelnen Schritten der Diagnostik und Therapie, sondern vielmehr in deren Koordinierung.

\section{Strukturelle Voraussetzungen}

Mit der Einführung von Traumanetzwerken durch den Arbeitskreis AKUT der Deutschen Gesellschaft für Unfallchirurgie (DGU) wird in Deutschland die Zuordnung sämtlicher Traumazentren in unterschiedliche Versorgungsstufen angestrebt (www.dgu-traumanetzwerk.de). Auch in Nordamerika sind die Anforderungen an „trauma center“ durch das American College of Surgeons eindeutig festgelegt. Sie beinhalten neben entsprechenden personellen und apparativen Voraussetzungen auch die Durchführung von ATLS ${ }^{ø}$-Kursen (ATLS: „advanced trauma life support") und entsprechende Forschungsaktivitäten.

Während früher in der Literatur für den Schockraum eine Mindestgröße von
$30 \mathrm{~m}^{2}$ gefordert war, werden in neueren Publikationen $50 \mathrm{~m}^{2}$ in überregionalen Traumazentren empfohlen [8]. Seitens der Radiologie ist nicht nur die konventionelle Röntgendiagnostik, sondern auch der Betrieb eines Computertomographen (CT) $24 \mathrm{~h}$ am Tag sicherzustellen. Mutschler u. Kanz [18] forderten zudem die Durchführung der CT im Schockraum oder zumindest in unmittelbarer räumlicher Nähe [8, 18].

In einer Analyse bezüglich der apparativen Ausstattung in deutschen Schockräumen zeigte sich, dass lediglich 8 von 51 Kliniken über ein CT im Schockraum verfügen [3]. Das während der initialen Diagnostik häufig notwendige Umlagern des Patienten bedeutet nicht nur einen zusätzlichen Zeitverlust, sondern birgt auch das Risiko der Dislokation von Drainagen und Kathetern mit einer potenziellen Gefährdung des Patienten. Von verschiedenen Herstellern werden mittlerweile Transportsysteme angeboten, die ein häufiges Umlagern des Patienten im Rahmen der Diagnostik nicht mehr erfordern.

Zusätzliche radiologische Diagnoseverfahren wie Sonographie und Magnetresonanztomographie (MRT) sollten in einem Traumazentrum genauso verfügbar sein wie die Möglichkeit zur Intervention.

\section{Personelle Voraussetzungen}

In den zurzeit aktuellen Leitlinien zum Schockraummanagement gibt es in Abhängigkeit der Versorgungsstufe konkrete Empfehlungen zur Zusammensetzung des Teams [8]. Ein entsprechendes Beispiel für ein regionales Traumazentrum ist in - Tab. 1 dargestellt.

Im Einzelnen hat das versorgende Team unabhängig von seiner Zusammensetzung folgende Aufgaben:

- Fortführung der präklinisch begonnenen Therapie

- Schnelle Diagnose relevanter Verletzungen

- Prioritätenorientierte Behandlung

- Festlegung der weiteren Versorgung

\section{Unfallchirurgie}

Die Anwesenheit eines traumatologischen Oberarztes während der initialen Diagnostik und Behandlungsphase ist notwendig, um die Dauer der Schockraumphase und die Zeit bis zur Erstversorgung im Operationssaal zu verkürzen; ein Einfluss auf die Dauer der intensivmedizinischen Behandlung oder die Mortalität konnte jedoch nicht nachgewiesen werden [12]. Nach aktueller Datenlage ist diesbezüglich die Anwesenheit eines traumatologischen Oberarztes innerhalb von 20 min ausreichend $[2,12]$, wenn zu jeder Zeit die umgehende Anwesenheit eines Facharztes/“senior doctor , sichergestellt ist [16]. Nach unserer Erfahrung ist jedoch die permanente Anwesenheit eines traumatologischen Oberarztes wünschenswert. Zudem übernimmt der unfallchirurgische Oberarzt im deutschsprachigen Raum häufig während der Schockraumphase die Führung. 


\section{Anästhesiologe}

Seitens der anästhesiologischen Betreuung schwerstverletzter Patienten ist in einem überregionalen Traumazentrum die ständige Anwesenheit eines Anästhesisten mit Facharztstandard mit einem Oberarzt im Hintergrund gefordert [8]. Unserer Erfahrung nach hat sich jedoch auch die 24-stündige Anwesenheit eines anästhesiologischen Oberarztes z. B. bei schweren Mittelgesichtsverletzungen bewährt.

Seitens der Anästhesie werden Aufgaben, die der Kontrolle und Stabilisierung von Vitalfunktionen dienen, aber auch die Einleitung bzw. Fortführung der Narkose im Rahmen der klinischen Erstversorgung wahrgenommen. Konkret beinhaltet das auch das Freihalten der Atemwege und die Sicherstellung einer ausreichenden Ventilation.

Die kontinuierliche Infusion kristalloider und kolloidaler Lösungen oder von Blutkomponenten fällt ebenfalls in den Bereich der Anästhesie. Ist nach der präklinischen Versorgung keine ausreichende Anzahl großlumiger venöser Zugänge vorhanden, ist der Anästhesist für deren Anlage ebenso verantwortlich wie für die von zentral-venösen und arteriellen $\mathrm{Zu}$ gängen, um ein erweitertes hämodynamisches Monitoring durchzuführen.

Die Kommunikation zwischen Anästhesie und Chirurgie spielt eine entscheidende Rolle, da Probleme im Bereich der Atemwege, der Ventilation und Perfusion von zentraler Relevanz bei der weiteren Diagnostik und Therapie sind.

\section{Radiologen}

Bezüglich ihrer Einbindung ist die Datenlage widersprüchlich. Während einige Studien eine adäquate Beurteilung von konventionellen Röntgenbildern und sogar der Schnittbilddiagnostik durch Nichtradiologen zeigen konnten [28], favorisieren andere Untersuchungen eine Beurteilung der Bildgebung durch Radiologen $[6,27]$. Die Anwesenheit eines radiologischen Oberarztes scheint dagegen nicht erforderlich zu sein $[4,25]$.

Trauma Berufskrankh 2010 - 12 [Suppl 4]:416-420 DOI 10.1007/s10039-010-1636-5

C) Springer-Verlag 2010

\section{Frink - W. Koppert · H. Andruszkow · C. Krettek · F. Hildebrand Schnittstelle Schockraummanagement}

\section{Zusammenfassung}

Die Behandlung polytraumatisierter Patienten ist nach wie vor eine Herausforderung. Die Schockraumbehandlung stellt das Bindeglied zwischen präklinischer und klinischer Versorgung dar. Ihr Ziel sind v. a. die schnelle Diagnose und prioritätenorientierte Behandlung lebensbedrohlicher Verletzungen. Empfehlungen bezüglich der für eine adäquate Versorgung im Schockraum unverzichtbaren strukturellen sowie personellen Voraussetzungen in Abhängigkeit der Versorgungsstufe des Krankenhauses gibt das Weißbuch Schwerverletztenversorgung der Deutschen Gesellschaft für Unfallchirurgie (DGU). Diese Empfehlungen können in Abhängigkeit der jeweiligen Infrastruktur und des vorliegenden Verletzungsmuster erweitert werden. Eine optimale Versorgung des Patienten ist nur durch die Zusammenarbeit in einem eingespielten Team und ein kooperatives und kollegiales Handeln der einzelnen Beteiligten unter Leitung eines erfahrenen Vertreters der Unfallchirurgie/Chirurgie bzw. Anästhesie zu gewährleisten. Entsprechende Ausbildungskonzepte, wie ATLS ${ }^{\circledast}{ }_{\text {, }}$ advanced trauma life support"), können helfen, einen Behandlungsalgorithmus zu etablieren. Ein inund externes Qualitätsmanagement kann die Effizienz der Behandlung steigern.

\section{Schlüsselwörter}

Polytrauma - Lebensbedrohliche Verletzung . Schockraumbehandlung - Weißbuch Schwerverletztenversorgung · Advanced Trauma Life Support (ATLS ${ }^{\circledast}$ )

\section{Interface: resuscitation room management}

\section{Abstract}

Treatment of patients with multiple injuries has improved in recent years but still remains a major challenge for the treating team. The treatment in the emergency room represents the interface between prehospital and clinical trauma care. Management of patients with multiple injuries in the emergency room aims at a rapid diagnosis and treatment of life-threatening injuries. Adequate trauma care requires not only structural but also human resources. Recommendations are given by the Deutsche Gesellschaft für Unfallchirurgie (DGU) for all hospitals. The cooperation of a well-rehearsed trauma team is necessary to guarantee optimal trauma care. Special training concepts [i.e. Advanced Trauma Life Support (ATLS) ${ }^{\oplus}$ ] can help to establish treatment algorithms. An internal and external quality management can increase the efficiency of the treatment of patients with multiple injuries.

\section{Keywords}

Multiple injuries - Life-threatening injury . Trauma room treatment . White Paper on management of severely injured patients . Advanced Trauma Life Support (ATLS ${ }^{\oplus}$ ) 


\begin{tabular}{|c|c|}
\hline \multirow[t]{8}{*}{ Basisschockraumteam } & $\begin{array}{l}1 \text { Facharzt für Orthopädie/Unfallchirurgie mit Zusatzweiterbildung } \\
\text { Spezielle Unfallchirurgie }\end{array}$ \\
\hline & $\begin{array}{l}1 \text { Weiterbildungsassistent in Orthopädie/Unfallchirurgie } \\
\text { oder } \\
1 \text { Weiterbildungsassistent in Zusatzweiterbildung Spezielle Unfallchirurgie } \\
\text { oder } \\
1 \text { Weiterbildungsassistent in Viszeral- oder Allgemeine Chirurgie }\end{array}$ \\
\hline & 1 Facharzt oder Weiterbildungsassistent für Anästhesiologie \\
\hline & 1 Facharzt oder Weiterbildungsassistent für Radiologie \\
\hline & 2 Pflegekräfte Chirurgie \\
\hline & 1 Pflegekraft Anästhesiologie \\
\hline & 1-mal medizinisch-technisches Radiologiepersonal \\
\hline & 1-mal Transportpersonal \\
\hline \multirow[t]{5}{*}{ Hintergrund } & $\begin{array}{l}1 \text { Facharzt für Orthopädie/Unfallchirurgie mit Zusatzweiterbildung } \\
\text { Spezielle Unfallchirurgie (Oberarzt) }\end{array}$ \\
\hline & 1 Facharzt für Viszeralchirurgie oder Allgemeinchirurgie (Oberarzt) \\
\hline & 1 Facharzt für Anästhesiologie (Oberarzt) \\
\hline & 1 Facharzt für Radiologie (Oberarzt) \\
\hline & 2 Operationspflegekräfte \\
\hline \multirow{11}{*}{$\begin{array}{l}\text { Erweitertes Schock- } \\
\text { raumteam (Anwesen- } \\
\text { heit innerhalb eines } \\
\text { adäquaten Zeitraumes) }\end{array}$} & Facharzt für Neurochirurgie \\
\hline & Facharzt für Gefäßchirurgie \\
\hline & Facharzt für Thoraxchirurgie \\
\hline & Facharzt für Mund-Kiefer-Gesichts-Chirurgie \\
\hline & Facharzt für HNO \\
\hline & Facharzt für Augenheilkunde \\
\hline & Facharzt für Urologie \\
\hline & Facharzt für Gynäkologie \\
\hline & Facharzt für Plastische Chirurgie \\
\hline & Facharzt für Pädiatrie \\
\hline & Facharzt für Kinderchirurgie \\
\hline
\end{tabular}

\section{Neurochirurg}

Bei Verdacht auf intrakranielle Verletzungen sollte ein Vertreter der Neurochirurgie zum Schockraumteam gehören. Nach aktueller Datenlage ist die initiale Anwesenheit zwar nicht erforderlich, dennoch sollte er innerhalb von 30 min therapeutisch eingreifen können [17].

Die Behandlung Schädel-Hirn-traumatisierter Patienten durch einen neurotraumatologisch erfahrenen Chirurgen zeigte gute Ergebnisse [20]. Jedoch wird für ein überregionales Traumazentrum im Weißbuch Schwerstverletztenersorgung der DGU [8] die 24-stündige Anwesenheit eines Facharztes für Neurochirurgie gefordert.

\section{Pädiater/pädiatrischer Chirurg}

Die Behandlung schwerstverletzter Kinder ist nicht nur medizinisch eine $\mathrm{He}$ -

\section{Faktor Zeit}

Auch nach dem initialen Insult schreitet die Schädigung des Organismus aufgrund des Gewebedefekts und konsekutiv ausgeschütteter Mediatoren voran. Insbesondere die limitierten Kompensationsmechanismen machen die Zeit zu einem entscheidenden Faktor bei der Behandlung polytraumatisierter Patienten, was bereits relativ früh mit dem Ausdruck "golden hour of shock“ durch Cowley u. Dunham [7] belegt wurde. Die angegebene Zeitspanne ist zwar nach den heutigen Erkenntnissen nicht mehr aktuell, jedoch steht dieser Begriff noch immer beispielhaft für die Zeitspanne, die bis zur Erstversorgung polytraumatisierter Patienten nicht verstreichen darf.

In verschiedenen Analysen der präklinischen Versorgungszeit wurde klar, dass eine nicht unerhebliche Zeitspanne bereits bei der Ankunft des Patienten in der Klinik verstrichen ist. Probleme, die bei der Versorgung akut oder perakut auftreten (z. B. Spannungspneumothorax), haben ihren Ursprung in diesem vorklinischen Intervall. Dasselbe gilt für die Langzeitkomplikationen ["adult respiratory distress syndrom“ (ARDS), Nierensuffizienz, usw.], zu denen es während der intensivmedizinischen Betreuung kommen kann. So wurde z. B. für hämodynamisch instabile Patienten mit perforierenden Verletzungen des Abdomens eine Steigerung der Letalität von $1 \%$ pro 3 min bis zum Beginn der Laparotomie gezeigt [5].

Aus Gründen der Zeitersparnis sind diagnostische und therapeutische Schritte in Zentren mit der entsprechenden Infrastruktur eng miteinander verbunden und werden parallel durchgeführt. Entsprechende Ausbildungskonzepte, wie ATLS ${ }^{\circledast}$, berücksichtigen dies in ihren Inhalten.

Entsprechend der aktuellen Studienlage sollte das Schockraumteam den Patienten bereits bei dessen Ankunft erwarten [21]. Bewährt hat sich dabei die Benachrichtigung der Mitglieder über spezielle Funker, die zeitgleich das gesamte Team alarmieren [30].

Unter der Prämisse „treat first what kills first" hängt die Reihenfolge der durchzuführenden Maßnahmen streng von den Vitalparametern und vom Ver- 
letzungsmuster des Patienten ab. Die Planung der weiteren Therapie wird entscheidend von den endgültigen Diagnosen beeinflusst. Aus diesem Grund wird seit einigen Jahren die CT als primäre radiologische Diagnostik statt der konventionellen Röntgendiagnostik diskutiert. Die zeitnahe Durchführung einer Ganzkörper-CT im Schockraum konnte dabei die Zeit bis zur Stellung der Diagnosen um 25 min verkürzen [31]. Demgegenüber steht das Risiko, während dieser Zeitspanne keine notfallmäßigen Interventionen durchführen zu können.

Bei der Erstellung eines entsprechenden Schockraumalgorithmus ist die jeweilige Infrastruktur von Bedeutung und sollte entsprechend berücksichtigt werden [18].

\section{Horizontale Kommunikation und Führung eines Traumateams}

Eine adäquate Versorgung schwerstverletzter Patienten ist nur dann möglich, wenn sich alle Beteiligten der Behandlung des Patienten voll verschreiben. Daraus wird deutlich, dass sich zwischen allen beteiligten Disziplinen und auch dem Pflegepersonal ein Teamgeist entwickeln muss. Voraussetzung dafür sind aber nicht nur das gegenseitige Vertrauen und Verständnis, sondern auch eine auf Kooperation und Kollegialität basierende Kommunikation. Beim Umgang zwischen den einzelnen beteiligten Disziplinen wird der Begriff der horizontalen Kommunikation verwendet, um zu verdeutlichen, dass Gleichgestellte kommunizieren [18].

Im Rahmen der Schockraumbehandlung wird es jedoch zwangsläufig immer wieder zu Situationen kommen, in denen einzelne Mitglieder des Teams gegensätzlicher Meinung sind. In diesem Fall ist es wichtig, dass von einem Teamleiter nach Absprache mit den beteiligten Fachdisziplinen eine Entscheidung getroffen wird. In mehreren Studien wurde die Notwendigkeit eines solchen verantwortlichen Arztes während der Schockraumphase gezeigt. Unter einem „team leader" wurde nicht nur die Zeit für die körperliche Untersuchung, sondern auch die Festlegung weiterer therapeutischer Schritte verkürzt [13]. Stellvertretend für andere Verletzungen konnte bei Patienten mit Schädelverletzungen oder Femurfrakturen die Zeit bis zum Beginn der operativen Therapie durch einen Verantwortlichen im Schockraum gesenkt werden [1]. Bedingt durch die unterschiedlichen Ausbildungssysteme kann der verantwortliche Arzt aus dem chirurgisch/traumatogischen Gebiet, aus dem Department für Emergency Medicine oder der Anästhesiologie kommen.

Unabhängig von der stellenden Fachrichtung sind durch den verantwortlichen Arzt während der Schockraumphase folgende Aufgaben zu erfüllen:

- Übernahme des Patienten vom erstbehandelnden Kollegen

- Durchführung und Überwachung der therapeutischen und diagnostischen Maßnahmen

- Konsultation anderer Disziplinen

- Koordinierung der einzelnen therapeutischen und diagnostischen Maßnahmen über die Schockraumphase hinaus

- Festlegung der weiteren Behandlungsstrategie unter Berücksichtigung der anderen Fachdisziplinen [10]

\section{Qualitätssicherung}

\section{Konflikte}

Neben der fachlichen Kompetenz der einzelnen Mitglieder des Traumateams ist die „team performance“ ein in letzter Zeit ins Zentrum der Betrachtung gerückter Faktor. Eine objektive Betrachtung und Bewertung der Schockraumbehandlung wäre über eine Videodokumentation möglich, diese ist jedoch im Einzelfall häufig aus juristischen oder personalpolitischen Gründen nicht etablierbar.

In einer Studie aus der Schweiz wurde mittels Mitarbeiterbefragung eine Qualitätskontrolle des Schockraummanagements durchgeführt. Dabei zeigte sich in 18,6\% der Fälle ein Konflikt, an dem in der Mehrzahl der Fälle ein Vertreter der Chirurgie beteiligt war. In 1/3 der Fälle wurde durch Beteiligte ein ungenügendes Zeitmanagement kritisiert. Die Beurteilung der Gesamtqualität hing nicht nur von der Fachdisziplin ab, sondern auch von der Ausbildungsstufe: Chirurgie und Radiologie beurteilten die Qualität höher als Anästhesisten; Assistenzärzte stuften die
Qualität geringer ein als Ärzte mit Leitungsfunktion. Mit höherer Verletzungsschwere verschlechterte sich die Beurteilung hinsichtlich Zeitmanagement, Gesamtablauf und eigener Zufriedenheit [11].

\section{Prozessqualität}

Unter objektiven Gesichtspunkten kommt es bei jeder Schockraumversorgung zu Fehlern, die jedoch in der deutlichen Mehrzahl der Fälle keine negativen Konsequenzen für den Patienten haben. Um systematische Fehler zu entdecken und damit das Management der Polytraumaversorgung $\mathrm{zu}$ verbessern, wurden schon früh die Einführung und Weiterentwicklung von Versorgungsstandards und eines Qualitätsmanagements gefordert. Bei der Integration von Versorgungsstandards kann auch die Teilnahme der einzelnen Beteiligten an entsprechenden Kurssystemen wie ATLS ${ }^{\varpi}$-Schulungen helfen.

In der aktuellen Literatur finden sich zahlreiche Hinweise, dass die Einführung eines entsprechend den Möglichkeiten der Klinik angepassten Schockraumkonzepts zu einer Verbesserung der Behandlung führt [9, 22, 24]. Trotzdem sind Versäumnisse und Fehler in dieser Versorgungsphase nicht auszuschließen. In einer aktuellen Untersuchung aus den USA wurde in fast $10 \%$ der Behandlungsfälle (76/764) von verstorbenen Polytraumapatienten ein Fehler in der Schockraumphase gefunden, der bei 16 Patienten (2\%) einen so genannten „preventable death“ zur Folge hatte. Die weitaus größere zum Tode führende Fehlerhäufigkeit fand sich in Patienten mit einem ISS („injury severity score") von 1-15 (20\%) und 16-24 ( 16\%) und weniger in Patienten mit einem ISS $>25$ ( 8\%) [14].

Für das interne Qualitätsmanagement gibt es verschiedene Ansätze. Eine Steigerung der Effizienz und Qualitätssteigerung ließ sich u. a. durch das quartalsweise Abhalten eines interdisziplinären Qualitätszirkels Schockraum erreichen, in dessen Rahmen Behandlungsstandards erstellt und abgeschlossene Fälle im Nachhinein besprochen wurden [23].

Bereits 1993 wurde durch die DGU das Traumaregister als Instrument zur Durchführung eines externen Qualitätsmanage- 
ments eingeführt. Zunächst müssen von allen beteiligten Kliniken Daten von behandelten Schwerstverletzten übermittelt werden. Diese werden auf ihre Plausibilität geprüft und ausgewertet. Den teilnehmenden Kliniken wird am Jahresende ein anonymisierter Bericht zur Verfügung gestellt, aus dem die Parameter der eigenen Klinik im Verhältnis zu den anderen Kliniken ersichtlich sind. Laut einer Studie führte dieses Vorgehen in $85 \%$ der teilnehmenden Kliniken zur Einführung qualitätsverbessernder Maßnahmen [22].

\section{Fazit für die Praxis}

Für die adäquate Versorgung schwerstverletzter Patienten ist ein perfektes Schockraummanagement von zentraler Bedeutung. Ziel der Behandlung sind die Stabilisierung des Patienten, das Diagnostizieren relevanter Verletzungen und die prioritätenorientierte Behandlung. Ein ggf. zu ergänzendes Basisschockraumteam, bestehend aus Vertretern der Unfallchirurgie/Chirurgie und Anästhesie sowie entsprechenden Pflegekräften, muss dabei großen Ansprüchen genügen und unter Stress prognostisch relevante Entscheidungen treffen. Voraussetzung dafür sind Kommunikationsbereitschaft und -fähigkeit der Beteiligten. Maßnahmen zur internen und externen Qualitätssicherung können die Effizienz der Behandlung verbessern.

\section{Korrespondenzadresse}

\section{PD Dr. M. Frink}

Unfallchirurgische Klinik,

Medizinische Hochschule Hannover, Carl-Neuberg-Straße 1, 30625 Hannover frink.michael@MH-Hannover.de

Interessenkonflikt. Keine Angaben

\section{Literatur}

1. Alberts KA, Bellander BM, Modin G (1999) Improved trauma care after reorganisation: a retrospective analysis. Eur J Surg 165(5):426-430

2. Allen DM, Hicks G, Bota GW (1998) Outcomes after severe trauma at a northern Canadian regional trauma centre. Can J Surg 41(1):53-58

3. Beck A, Bischoff M, Gebhard F et al (2004) Diagnostics and equipment in the shock trauma room. Unfallchirurg 107(10):862-870
4. Chung JH, Strigel RM, Chew AR et al (2009) Overnight resident interpretation of torso $\mathrm{CT}$ at a level 1 trauma center - an analysis and review of the literature. Acad Radiol 16(9):1155-1160

5. Clarke JR, Trooskin SZ, Doshi PJ et al (2002) Time to laparotomy for intra-abdominal bleeding from trauma does affect survival for delays up to $90 \mathrm{mi}-$ nutes. J Trauma 52(3):420-425

6. Compere V, Genevois A, Le Corre A et al (2003) Influence of medical speciality and experience on interpretation of helicoidal thoracic computed tomography in blunt chest trauma. Intensive Care Med 29(5):770-773

7. Cowley RA, Dunham CM (1982) Shock trauma/critical care manual. Initial assessment and management. University Park Press, Baltimore

8. Deutsche Gesellschaft für Unfallchirurgie (2006) Weißbuch Schwerverletzten-Versorgung. DGU, Berlin

9. Dutton RP, Stansbury LG, Leone S et al (2010) Trauma mortality in mature trauma systems: are we doing better? An analysis of trauma mortality patterns, 1997-2008. J Trauma Jan 20. [Epub ahead of print]

10. Frink M, Probst C, Krettek C, Pape HC (2007) Clinical management of polytraumatized patients in the emegergency room - duty and assignment of the trauma surgeon. Zentralbl Chir 132(1):49-53

11. Gross T, Amsler F, Ummenhofer W et al (2005) Interdisciplinary emergency room management of trauma patients from the standpoint of coworkers. Chirurg 76(10):959-966

12. Helling TS, Nelson PW, Shook JW et al (2003) The presence of in-house attending trauma surgeons does not improve management or outcome of critically injured patients. J Trauma 55(1):20-25

13. Hoff WS, Reilly PM, Rotondo MF et al (1997) The importance of the command-physician in trauma resuscitation. J Trauma 43(5):772-777

14. Ivatury RR, Guilford K, Malhotra AK et al (2008) Patient safety in trauma: maximal impact management errors at a level I trauma center. J Trauma 64(2):265-270

15. Knudson MM, Shagoury C, Lewis FR (1992) Can adult trauma surgeons care for injured children? J Trauma 32(6):729-737

16. Lecky F, Woodford M, Yates DW (2000) Trends in trauma care in England and Wales 1989-1997. UK Trauma Audit and Research Network. Lancet 355(9217):1771-1775

17. Lucas CE, Dombi GW, Crilly RJ et al (1997) Neurosurgical trauma call: use of a mathematical simulation program to define manpower needs. J Trauma 42(5):818-823

18. Mutschler W, Kanz KG (2002) Interdisciplinary shock room management: responsibilities of the radiologist from the trauma surgery viewpoint. Radiologe 42(7):506-514

19. Nakayama DK, Copes WS, Sacco W (1992) Differences in trauma care among pediatric and nonpediatric trauma centers. J Pediatr Surg 27(4):427431

20. Parzhuber $A$, Wiedemann $E$, Richter-Turtur $M$ et al (1994) The contribution of the general and trauma surgeon in neurotraumatology: experiences and results of 10 years. Unfallchirurg 97(12):615-618

21. Petrie D, Lane P, Stewart TC (1996) An evaluation of patient outcomes comparing trauma team activated versus trauma team not activated using TRISS analysis. Trauma and Injury Severity Score. J Trauma 41(5):870-873

22. Ruchholtz $S$ (2004) External quality management in the clinical treatment of severely injured patients. Unfallchirurg 107(10):835-843
23. Ruchholtz S, Waydhas C, Aufmkolk M et al (2001) Interdisciplinary quality management in the treatment of severely injured patients. Validation of a QM system for the diagnostic and therapeutic process in early clinical management. Unfallchirurg 104(10):927-937

24. Ruchholtz S, Waydhas C, Lewan U et al (2002) A multidisciplinary quality management system for the early treatment of severely injured patients: implementation and results in two trauma centers. Intensive Care Med 28(10):1395-1404

25. Ruchman RB, Jaeger J, Wiggins EF III et al (2007) Preliminary radiology resident interpretations versus final attending radiologist interpretations and the impact on patient care in a community hospital. AJR Am J Roentgenol 189(3):523-526

26. Segui-Gomez M, Chang DC, Paidas CN et al (2003) Pediatric trauma care: an overview of pediatric trauma systems and their practices in 18 US states. J Pediatr Surg 38(8):1162-1169

27. Velmahos GC, Fili C, Vassiliu P et al (2001) Aroundthe-clock attending radiology coverage is essential to avoid mistakes in the care of trauma patients. Am Surg 67(12):1175-1177

28. Vorhies RW, Harrison PB, Smith RS, Helmer SD (2002) Senior surgical residents can accurately interpret trauma radiographs. Am Surg 68(3):221225

29. Wachtel TL, Coniglio R, Bourg P et al (2001) The synergistic relationship between a level I trauma center and a regional pediatric trauma center. Semin Pediatr Surg 10(1):38-43

30. Wong K, Petchell J (2003) Trauma teams in Australia: a national survey. ANZ J Surg 73(10):819-825

31. Wurmb TE, Fruhwald $P$, Hopfner W et al (2009) Whole-body multislice computed tomography as the first line diagnostic tool in patients with multiple injuries: the focus on time. J Trauma 66(3):658665 